\title{
Directivity of Uniformly Spaced Optimum Endfire Arrays With Equal Sidelobes
}

\author{
M. T. Ma \\ Central Radio Propagation Laboratory, National Bureau of Standards, Boulder, Colo.
}

(Received March 5, 1965; revised April 16, 1965)

\begin{abstract}
A mathematical formulation is devised for a uniformly spaced and uniformly progressively phased antenna array of a given number of isotropic elements such that the final radiation pattern is endfire with no large backlobe. The pattern also has the maximum number of sidelobes of equal level, modifying the result of an earlier paper, even when the element spacing is made arbitrarily small. The directive gain, the first null beamwidth, the half-power beamwidth, the current excitations, and the required phases for such an array are given in curves as a function of the element spacing. It is shown that the limiting radiation characteristics when the element spacing approaches zero can also be determined in a relatively simple manner.
\end{abstract}

\section{Introduction}

Since the publication of Dolph's paper [1946], many articles dealing with the optimization between the beamwidth and the sidelobe level of linear antenna arrays have appeared in the literature. In summary, different types of mathematical formulations have been used by different investigators for different arrays, depending on whether the array is broadside or endfire, whether the total number of elements in the array is odd or even, and whether the element spacing is greater or less than one-half wavelength [Dolph, 1946; Riblet, 1947; DuHamel, 1953; Pritchard, 1955]. A unified approach covering all these cases was described by Ma and Cheng [196la].

The problem of maximizing the directivity of an array was investigated by Uzkov [1946]. He showed, by means of an orthogonal transformation in vector space, that the maximum directivity of an endfire array of $n$ isotropic elements, as the element spacing approaches zero, is numerically equal to $n^{2}$. Other techniques for improving directivities of endfire arrays were also proposed [Hansen and Woodyard, 1938; Goward, 1947]. Bloch, Medhurst and Pool [1953] investigated similar problems by using the impedance relation between the array elements, but only a very limited calculation was given in their paper. Based on their method, a rather extensive calculation of the maximum directivity of an endfire array of halfwave dipoles was made later [Stearns, 1965]. Recently, Tai made some investigation of the optimum directivity of uniformly spaced broadside arrays [1964] and the ordinary endfire arrays [1963] of isotropic sources or dipoles. The sidelobe level of an array was not considered or limited in any of these papers.

As far as the directivity of an array with equal sidelobes is concerned, Reuss [1959] derived a formula for the Chebyshev linear broadside arrays consisting of infinitesimal dipoles, but his formula provides no information concerning the effect on the directivity when the element spacing changes. Stegen [1960] obtained an expression for the directivity in terms of Chebyshev polynomials, but his expression is valid only when the element spacing is an integral multiple of a half-wavelength. A general approach for dealing with this problem outlined by Ma and Cheng [1961a] was based on the idea of keeping the number of sidelobes as great as possible and with a (desired) equal level even when the element spacing was less than onehalf wavelength. Pertinent numerical results on directive gains, however, were not reported there. More recently, while attempting to obtain some numerical result, it was discovered that, according to the procedures outlined by Ma and Cheng [1961a], the sidelobes in the final radiation pattern synthesized for an odd number of elements are not all equal in level, since there always is a higher sidelobe occurring near $\theta=\pi / 2$, and that the final pattern for an even number of elements is not physically realizable (e.g., the "power" pattern function will become negative for some physical angles). It is the purpose of this paper to modify the procedures given before in such a way that the final pattern will have all sidelobes at an equal level. The modified procedures given in this paper will be valid for both an odd and even number of elements.

\section{Mathematical Formulation}

The field from a linear array of $n$ equispaced elements as given in figure 1 can be mathematically represented by the polynomial:

$$
E(z)=\sum_{k=0}^{n-1} a_{k} z^{k}
$$

where

$$
z=\exp (j \psi)
$$




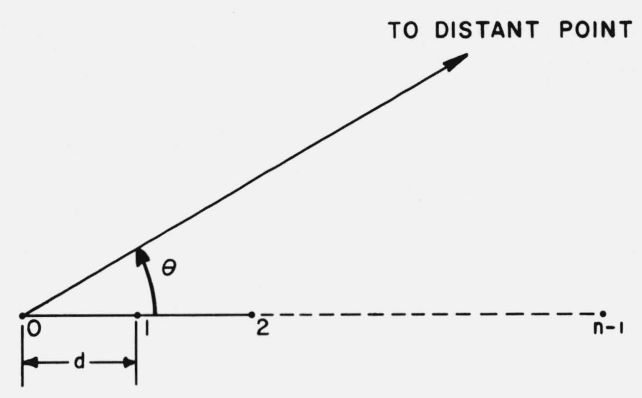

Figure 1. A linear array of $\mathrm{n}$ equi-spaced isotropic elements.

$$
\psi=\frac{2 \pi}{\lambda} d \cos \theta+\alpha
$$

and $a_{k}$ represents the excitation of the $k$ th element. In this paper $a_{k}$ are all real, implying that the array is uniform-progressively phased $(\alpha)$. It was previously shown [Cheng and Ma, 1960] that, by introducing a new variable, $y$,

$$
y \equiv z+z^{-1}=2 \cos \psi=2 \cos \left(\frac{2 \pi}{\lambda} d \cos \theta+\alpha\right)
$$

one can express $|E(z)|^{2}$, the power pattern, as a polynominal of $y$,

$$
\begin{aligned}
|E(z)|^{2} & =E(z) E\left(z^{-1}\right) \\
& =\left.\sum_{m=0}^{n-1} b_{m}\left(z^{m}+z^{-m}\right)\right|_{z+z^{-1}=y} \rightarrow P(y),
\end{aligned}
$$

where

$$
\begin{aligned}
& b_{m}=\sum_{i=0}^{n-1-m} a_{i} a_{m+i}, \quad 1 \leqslant m \leqslant(n-1), \\
& b_{0}=\frac{1}{2} \sum_{i=0}^{n-1} a_{i}^{2} .
\end{aligned}
$$

Since $P(y)$ is a polynominal of $(n-1)$ th degree with real coefficients, and is non-negative for all physical values of $y$ in the interval $[-2,2]$, it generally can only contain a combination of the following possible elementary factors:

(i) $\quad\left(y+b_{k}\right)$ or $\left(b_{k}-y\right)$ with $b_{k}$ real and $\geqslant 2$,

(ii) $\quad\left(y+b_{k}\right)^{2}$ with $b_{k}$ real and $\left|b_{k}\right|<2$,

(iii) $\quad\left(y^{2}+2 b_{k 1} y+b_{k 1}^{2}+b_{k 2}^{2}\right)$ with $b_{k 1}$ and $b_{k 2}$ real.

In particular, for arrays producing the maximum possible number $(n-1)$ of physical nulls in the visible range $-2 \leqslant y \leqslant 2$, the only factors that can appear in $P(y)$ are $(y+2)$ and $\left(y+b_{k}\right)^{2}$ with $\left|b_{k}\right|<2$. Therefore, depending on whether $n$ is odd or even, $P(y)$ for such an array must be:

$$
P_{0}(y)=\prod_{k=1}^{\frac{n-1}{2}}\left(y+b_{k}\right)^{2} \quad \text { for odd } n,
$$

$$
P_{\mathrm{e}}(y)=(y+2) \prod_{k=1}^{\frac{n-2}{2}}\left(y+b_{k}\right)^{2} \text { for even } n,
$$

where all $b_{k}$ are real, distinct, and $\left|b_{k}\right|<2$. In (8) and (9), we have assumed that $y=2$ is the location of the main beam. The corresponding array polynominals are, respectively,

$$
E_{0}(z)=\prod_{k=1}^{\frac{n-1}{2}}\left(1+b_{k} z+z^{2}\right) \quad \text { for odd } n
$$

and

$$
E_{\mathrm{e}}(z)=(1+z) \prod_{k=1}^{\frac{n-2}{2}}\left(1+b_{k} z+z^{2}\right) \text { for even } n .
$$

The excitation coefficients $a_{k}$ in (1) can then be determined by expanding (10) or (11).

With this preliminary analysis given, the general procedure of synthesizing an array having the maximum number of nulls can now be summarized as follows: (i) to determine $b_{k}$ for (8) or (9) according to a specification, and (ii) to determine the required excitation coefficients using (10) or (11).

\section{Directivity of Endfire Arrays With Equal Sidelobes}

For arrays having all the sidelobes equal in level, the locations of nulls $\left(y=-b_{k}\right)$ and sidelobes $\left(y=y_{l}\right)$ are related in a special manner, where

$$
\begin{aligned}
2>\left(-b_{1}\right)>y_{1} & >\left(-b_{2}\right)>y_{2}>\ldots \\
& >y_{\frac{n-3}{2}}>\left(-\frac{b_{\frac{n-1}{2}}}{2}>(-2) \text { for odd } n,\right.
\end{aligned}
$$

and

$2>\left(-b_{1}\right)>y_{1}>\ldots>y_{\frac{n-2}{2}}>(-2)$ for even $n$.

The parameters $b_{k}$ in (8) or (9), the corresponding excitation coefficients $a_{k}$ in (1), and the location of sidelobes can be uniquely determined once a desired sidelobe level relative to the main beam or a desired first null location $\left(-b_{1}\right)$ is specified. The detailed derivations and procedures are omitted here, but can be found elsewhere [Ma and Cheng, 196lb].

After a power polynomial $P(y)$ is determined, the directivity or the directive gain of an endfire array with equal sidelobes can be calculated according to the conventional definition [Silver, 1949]:

$$
G=\frac{\left(\frac{4 \pi}{\lambda} d\right) P\left(y_{a}\right)}{W_{0}}
$$


where

$$
W_{0}=\int_{y_{a}}^{y_{b}} \frac{P(y)}{\sqrt{4-y^{2}}} d y
$$

with the limits given by

$$
\begin{aligned}
& y_{b}=2 \cos \psi_{b}=2 \cos \left(-\frac{2 \pi}{\lambda} d+\alpha\right) \text { when } \theta=\pi \\
& y_{a}=2 \cos \psi_{a}=2 \cos \left(\frac{2 \pi}{\lambda} d+\alpha\right) \quad \text { when } \theta=0 .
\end{aligned}
$$

Note that the polynominal $P(y)$ can always be rearranged in terms of $\left(4-y^{2}\right)$ such that the exact expression for $W_{0}$ (and hence for $G$ ) will be obtained by integrating (15) term by term.

\section{A Transformation When $d<\lambda / 2$}

To keep the discussion more specific, let us consider the $P_{0}(y)$ given in (8). The whole pattern for $d=\frac{\lambda}{2}$ (or $\frac{2 \pi}{\lambda} d=\pi$ ) is plotted as in figure 2 a when the ordinary endfire condition, $-\alpha=\frac{2 \pi}{\lambda} d=\pi$, is used. Note that, in this case, there always is a large backlobe at $\theta=\pi$ having the same magnitude as the main lobe at


FigURE 2. Power pattern of an array with equal sidelobes (odd $\mathrm{n}$ ).

$$
\begin{aligned}
& \text { (a) Ordinary endfire, }-\alpha=\frac{2 \pi}{\lambda} d=\pi \text {, } \\
& \text { (b) Optimum endfire, } d<d^{*}<\frac{\lambda}{2}, \alpha \neq 0,-\alpha \neq \frac{2 \pi}{\lambda} d \text {. }
\end{aligned}
$$

$\theta=0$. If a smaller $d$ is chosen, some portion of $P_{0}(y)$ in the left-hand side of figure $2 \mathrm{a}$ will be invisible. It is clear that there exists an element spacing $d^{*}<\frac{\lambda}{2}$ for the ordinary endfire array such that the whole visible region will be ended at $y^{*}=2 \cos \left(-\frac{4 \pi}{\lambda} d^{*}\right)$ and that the final pattern will have a sidelobe at $\theta=\pi$ having the same level as the other sidelobes. In this case, no modification for improving both the directivity and the beamwidth is possible if the sidelobe level remains unchanged. This actually is the case considered before by Rhodes [1953].

For $d<d^{*}$, some of the sidelobes will also be invisible, then only the visible portion of the pattern would be expanded into the region $0 \leqslant \theta \leqslant \pi$, making the beamwidth wider than the case when $d=d^{*}$. In order to make improvement under this circumstance, an optimum endfire condition $\alpha \neq-\frac{2 \pi}{\lambda} d$, together with a linear transformation of variables such as

$$
y^{\prime}=k_{1} y+k_{2}
$$

is devised such that the final pattern still remains much as it formerly did up to $y=y^{*}$. The parameters $k_{1}, k_{2}$ in (17), and $\alpha$ can be determined by the following conditions:

$$
\begin{aligned}
& y^{\prime}=2 \cos \left(\frac{2 \pi}{\lambda} d+\alpha\right) \quad \text { when } y=2 \quad(\theta=0), \\
& y^{\prime}=2 \cos \left(\frac{-2 \pi}{\lambda} d+\alpha\right) \text { when } y=y^{*} \quad(\theta=\pi), \\
& y^{\prime}=2 \quad \text { when } y=-2 .
\end{aligned}
$$

The first condition of (18) maps both the magnitude and location of the main lobe, the second condition assures that the maximum possible number of sidelobes at equal level be kept and that the large backlobe be avoided, and the third condition maps the sidelobe (an odd $n$ ) or the null (an even $n$ ) originally at $y=-2$ to $y^{\prime}=2$. The transformation (17) with the conditions given in (18) is shown in figure $2 \mathrm{~b}$. From this figure one can see that, for $d<d^{*}$, the transformation (17) always squeezes the whole pattern up to $y^{*}$ into the visible region and, therefore, helps to shift the pattern toward the main lobe, making the beamwidth narrower and the directivity higher. For this reason the transformed array is called the optimum endfire array. Solving (18), one obtains

$$
\begin{gathered}
\tan \frac{\alpha}{2}=\frac{2-\sqrt{2+y^{*}}}{2+\sqrt{2+y^{*}}} \tan \frac{\pi d}{\lambda}, \\
k_{1}=-\sin ^{2}\left(\frac{\alpha}{2}+\frac{\pi d}{\lambda}\right)<0, \\
k_{2}=2\left(1+k_{1}\right)=2 \cos ^{2}\left(\frac{\alpha}{2}+\frac{\pi d}{\lambda}\right)>0,
\end{gathered}
$$


where $y^{*}$ can be easily determined by the following simple relation:

$$
\begin{aligned}
& y^{*}=2\left(1-b_{(n+1) / 4}\right), \text { for } n \text { odd and } \frac{n-1}{2} \text { odd; } \\
& y^{*}=2\left(1+y_{(n-1) / 4}\right), \text { for } n \text { odd and } \frac{n-1}{2} \text { even, }
\end{aligned}
$$

or

$$
y^{*}=2+y_{1}-b_{(n-2) / 2} \text { for even } n .
$$

The phase $\alpha$ and the parameters $k_{1}$ and $k_{2}$ required to produce an optimum endfire array can be calculated from (19) once a $d<d^{*}$ is chosen. From the first equation of (19) it can be concluded that $\alpha$ is always located somewhere in the first quadrant in order to make a pattern of an endfire array optimum when $d<d^{*}$. Note that (19) also holds true when $d=d^{*}$ and yields $\alpha^{*}=\pi-\frac{2 \pi}{\lambda} d^{*}>0$. There seems to be a phase discontinuity of $(\pi)$ as compared with the phase required by the ordinary endfire condition. This extra phase of $\pi$ is actually taken care of by the fact that $k_{1}=-1$ and $k_{2}=0$ for the case of $d=d^{*}$. Of course, this apparent discrepancy can be removed if one tries to map $y=-2$ onto $y^{\prime}=-2$, replacing the third condition in (18). While this alternative transformation seems more natural, as far as the limiting case $d \rightarrow d^{*}$ is concerned, it will create a little mathematical difficulty in dealing with the limiting case to the other end $(d \rightarrow 0)$, as compared to what we have done [see (30) through (32)] by using the present approach. In any case, these two approaches yield the same array depending on whether one likes to have the final excitation coefficients $a_{k}$ alternating signs (as is clearly shown in fig. 6) or to have the sign reversal absorbed in $\alpha$ if using the alternative transformation suggested above. As pointed out before, it is not necessary to make further modification in phases for the endfire arrays with equal sidelobes when $d=d^{*}$ (considered by Rhodes [1953]) because the ordinary endfire condition for phase still holds for that particular situation. Also, since this paper is to primarily deal with cases when $d<d^{*}$, the aforementioned two alternative approaches would not make any difference as far as the presentation is concerned.

The optimization discussed above specifically for $P_{0}(y)$ applies as well to $P_{e}(y)$ given in (9).

From (17) one has

$$
y=k_{1}^{-1}\left(y^{\prime}-k_{2}\right)
$$

Substituting (21) into (8) and (9), the power polynomial becomes, respectively,

$$
Q_{0}\left(y^{\prime}\right)=k_{1}^{-(n-1)} \prod_{k=1}^{\frac{n-1}{2}}\left(y^{\prime}+b_{k}^{\prime}\right)^{2} \text { for odd } n,
$$

$$
\begin{aligned}
Q_{e}\left(y^{\prime}\right) & =k_{1}^{-(n-1)}\left(y^{\prime}-2\right) \prod_{k=1}^{\frac{n-2}{2}}\left(y^{\prime}+b_{k}^{\prime}\right)^{2} \\
& =\left|k_{1}^{-(n-1)}\right|\left(2-y^{\prime}\right) \prod_{k=1}^{\frac{n-2}{2}}\left(y^{\prime}+b_{k}^{\prime}\right)^{2} \text { for even } n,
\end{aligned}
$$

where

$$
b_{k}^{\prime}=k_{1} b_{k}-k_{2}
$$

Note that the second form of (23) is due to the fact that $k_{1}<0$, see (19), and $k_{1}^{-(n-1)}<0$ for an even $n$. It is then clear from this second form that $Q_{e}\left(y^{\prime}\right) \geqslant 0$ for $-2 \leqslant y^{\prime} \leqslant 2$, as it should be for a realizable power polynomial. Based on (22) and (23), the corresponding array polynomial becomes, respectively,

$$
\begin{array}{ll}
E_{0}^{\prime}(z)=\sqrt{k_{1}^{-(n-1)}} \prod_{k=1}^{\frac{n-1}{2}}\left(1+b_{k}^{\prime} z+z^{2}\right) & \text { for odd } n, \\
E^{\prime}(z)=\sqrt{\mid k_{1}^{-(n-1) \mid}}(1-z) \prod_{k=1}^{\frac{n-2}{2}}\left(1+b_{k}^{\prime} z+z^{2}\right) & \text { for even } n .
\end{array}
$$

A new set of excitation coefficients required for synthesizing the optimum endfire array can be determined by expanding (25) or (26).

The directivity $G^{\prime}$, the first null beamwidth $\left(2 \theta_{1}\right)$, and the half-power beamwidth $\left(2 \theta_{h}\right)$ can be calculated respectively from

$$
\begin{aligned}
G^{\prime} & =\frac{4 \pi \frac{d}{\lambda} Q\left(y_{a}^{\prime}\right)}{W_{0}^{\prime}}, \\
-b_{1}^{\prime} & =2 \cos \left(\frac{2 \pi}{\lambda} d \cos \theta_{1}+\alpha\right),
\end{aligned}
$$

and

$$
y_{h}^{\prime}=2 \cos \left(\frac{2 \pi}{\lambda} d \cos \theta_{h}+\alpha\right),
$$

where

$$
\begin{aligned}
& W_{0}^{\prime}=\int_{y_{a}^{\prime}}^{y_{b}^{\prime}} \frac{Q\left(y^{\prime}\right)}{\sqrt{4-y^{\prime 2}}} d y^{\prime}, \\
& y_{b}^{\prime}=2 \cos \left(-\frac{2 \pi}{\lambda} d+\alpha\right), \\
& y_{a}^{\prime}=2 \cos \left(\frac{2 \pi}{\lambda} d+\alpha\right),
\end{aligned}
$$

and $y_{h}^{\prime}$ is determined by

$$
Q\left(y_{h}^{\prime}\right)=\frac{1}{2} Q\left(y_{a}^{\prime}\right) .
$$






Figure 3. Directivities for optimum endfire arrays with equal sidelobes $(-20 \mathrm{~dB})$.

Numerical results, for $n=3$ through $n=7$ and for a sidelobe level $20 \mathrm{~dB}$ down from the main lobe, obtained by using these formulas are given in figures $3,4,5,6$, and 7 . For comparison, the corresponding directivities for various $d$, if the ordinary endfire condition $-\alpha=\frac{2 \pi}{\lambda} d$ is used throughout, are also included in figure 3 as the dashed curves. The points marked with $*$ on these dashed curves are those when $d=d^{*}$.

From figure $2 \mathrm{~b}$, it is seen that the smaller the $d$, the narrower will the beamwidth become, and the higher will the directivity be. In fact, the limiting radiation characteristics for $n=3,4$, and 5 when $d \rightarrow 0$, has also been obtained by taking the appropriate limiting process. Specifically, the equations in (19) have been replaced by (30) through (32), when $d \rightarrow 0$,

$$
\frac{\alpha}{2} \sim \frac{2-\sqrt{2+y^{*}}}{2+\sqrt{2+y^{*}}} \frac{\pi d}{\lambda}
$$

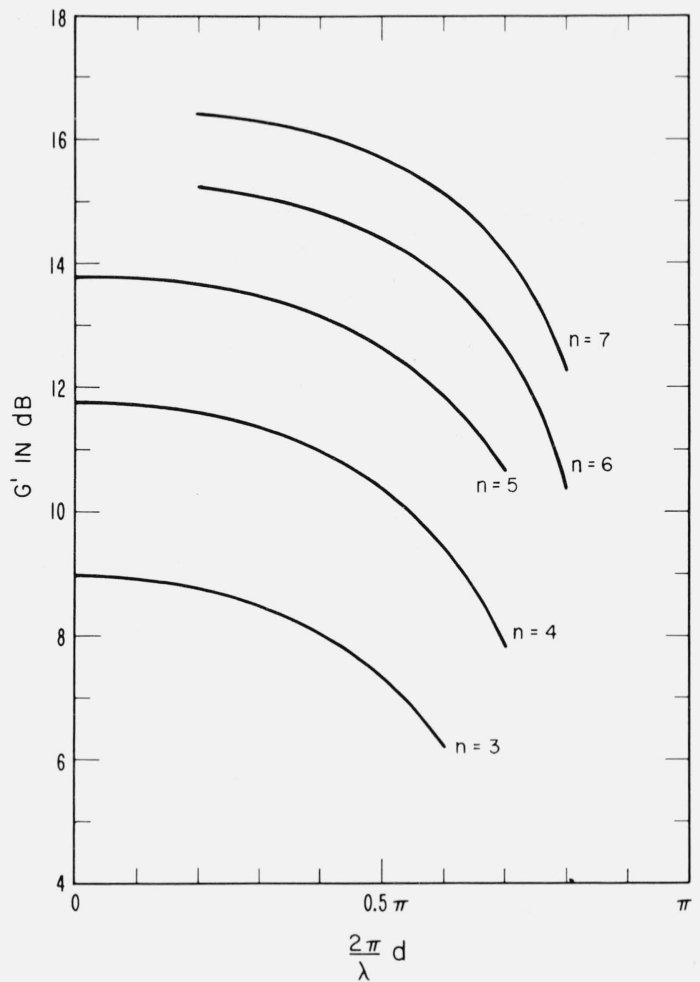

FIGURE 4. Directivities in $d B$ for optimum endfire arrays with equal sidelobes $(-20 \mathrm{~dB})$.

$$
\begin{aligned}
& k_{1} \sim-\left(x-\frac{x^{3}}{3 !}+\frac{x^{5}}{5 !}-\ldots\right)^{2}, \\
& k_{2} \sim 2\left(1-\frac{x^{2}}{2 !}+\frac{x^{4}}{4 !}-\ldots\right)^{2},
\end{aligned}
$$

where

$$
x=\frac{\alpha}{2}+\frac{\pi d}{\lambda} \sim \frac{4}{2+\sqrt{2+y^{*}}} \frac{\pi d}{\lambda} .
$$

The number of terms to be carried for $k_{1}$ and $k_{2}$ in (31) and (32) is determined by $n$, the total number of elements in the array.

\section{Conclusion}

A new way of calculating the directivity of a uniformly spaced endfire array with equal sidelobes, when the element spacing is made arbitrarily small, has been formulated. Numerical results on directivities, beamwidths, current excitations, and phases for a typical endfire array have been presented (figs. 3 through 7). The limiting radiation characteristics for $n=3,4$, and 5 when the element spacing approaches zero have also been included. An extended table for $n=3$ through $n=30$ with the sidelobe level varying from $10 \mathrm{~dB}$ through $50 \mathrm{~dB}$ below the main lobe is being prepared as an NBS monograph to be published soon. The investigation has been carried 


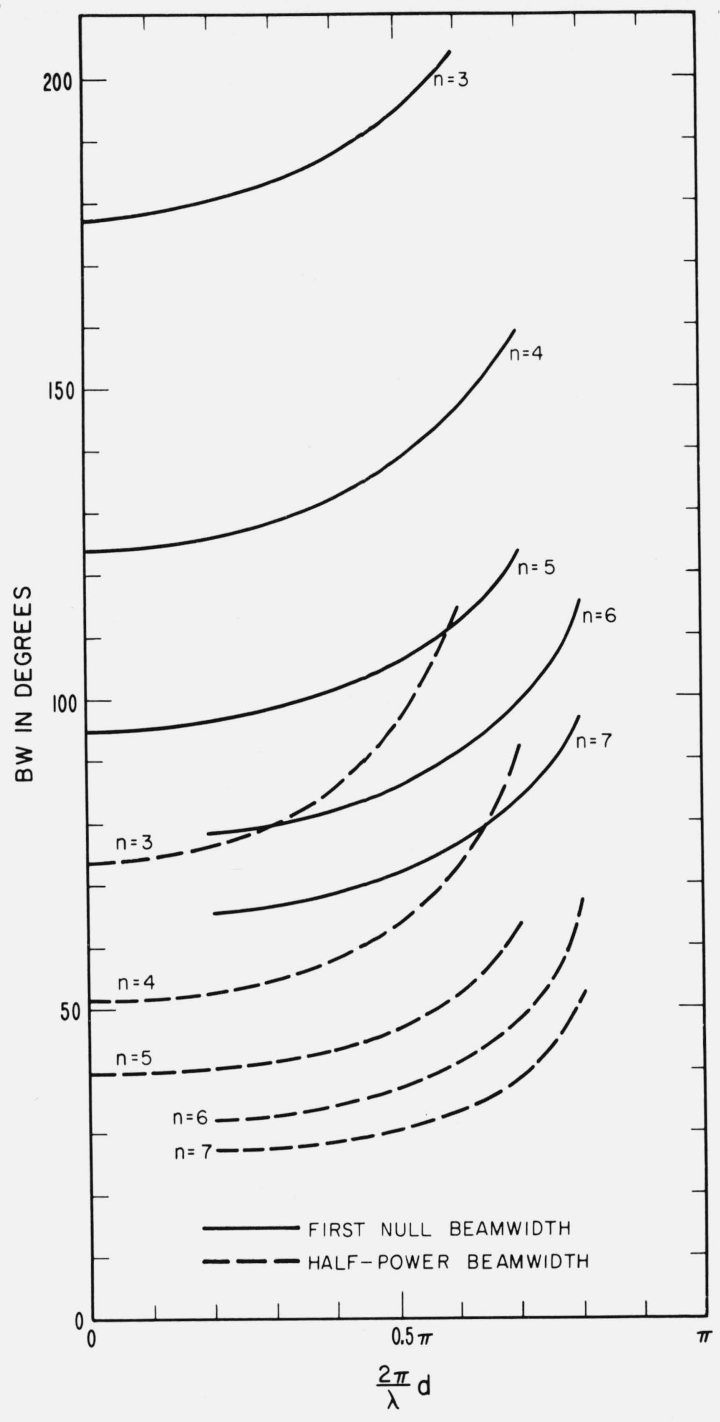

Figure 5. Beamwidths in degrees for optimum endfire arrays with equal sidelobes $(-20 \mathrm{~dB})$.

out only on a theoretical basis. The actual designing of this type of so-called supergain array is perhaps far more difficult in view of increased current magnitudes [Yaru, 1951], mutual couplings between elements, high ohmic losses, low efficiency, low effective radiation resistances [Wilmotte, 1948], critical mechanical tolerances, narrow bandwidth, and increased stored energy [Taylor, 1948].

The author thanks H. V. Cottony for his comments and for calling attention to some references, and A. C. Stewart for reading the manuscript.

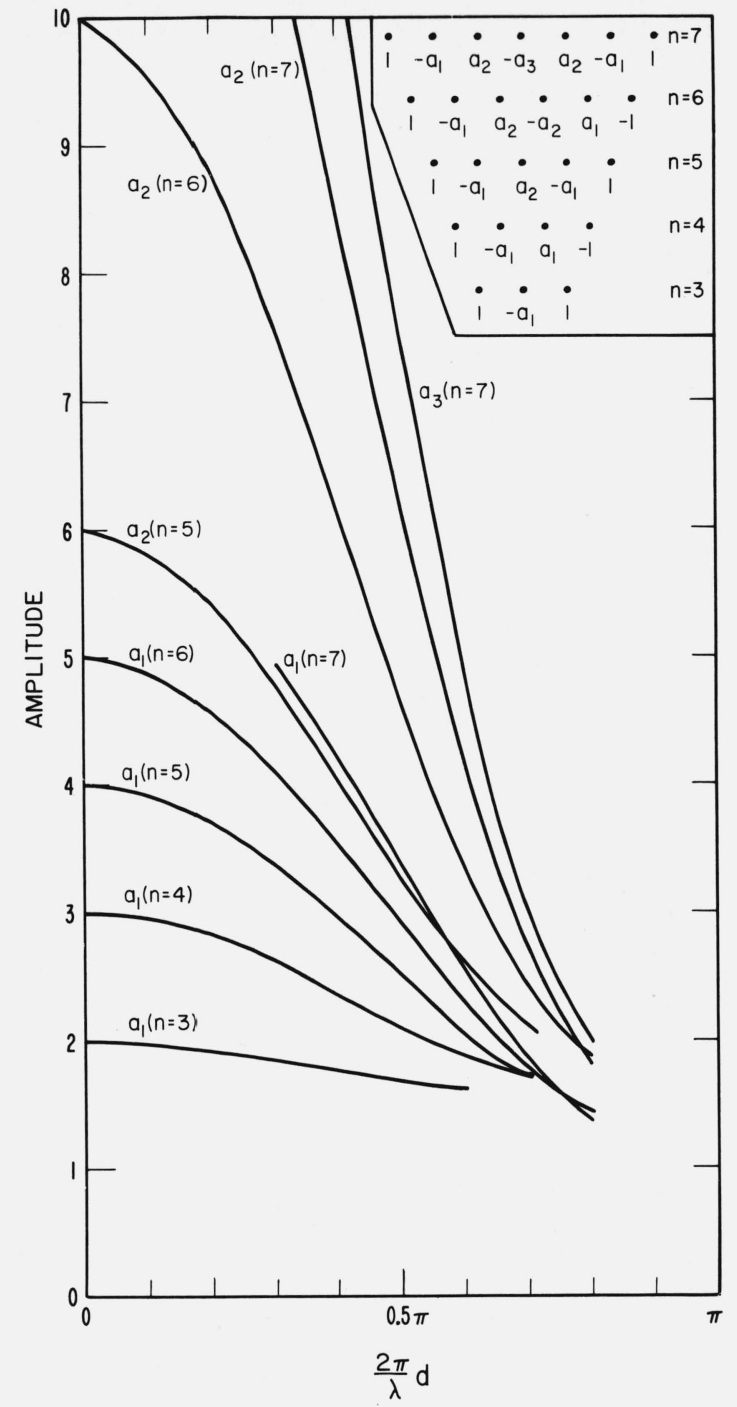

FIGURE 6. Excitation coefficients for optimum endfire arrays with equal sidelobes (-20 dB)

\section{References}

Bloch, A., R. G. Medhurst, and S. D. Pool (Sept. 1953), A new approach to the design of super-directive aerial arrays, J. Inst. Elec. Engrs. (London) 100 , part 3, 303-314.

Cheng, D. K., and M. T. Ma (May 1960), A new mathematical approach for linear array analysis, IEEE Trans. Ant. Prop. AP-8, 255-259.

Dolph, C. L. (June 1946), A current distribution for broadside arrays which optimizes the relationship between beamwidth and sidelobe level, Proc. IRE 34, 335-348.

DuHamel, R. H. (May 1953), Optimum patterns for endfire arrays, Proc. IRE 41, 652-659.

Goward, F. K. (Nov. 1947), An improvement in endfire arrays, J. Inst. Elec. Engrs. (London) 94, part 3, 415-418.

Hansen, W. W., and J. R. Woodyard (Mar. 1938), A new principle in directional antenna design, Proc. IRE 26, 333-345.

Ma, M. T., and D. K. Cheng (196la), A critical study of linear arrays with equal sidelobes, IRE Intern. Conv. Record 9, part 1, 110-122. 


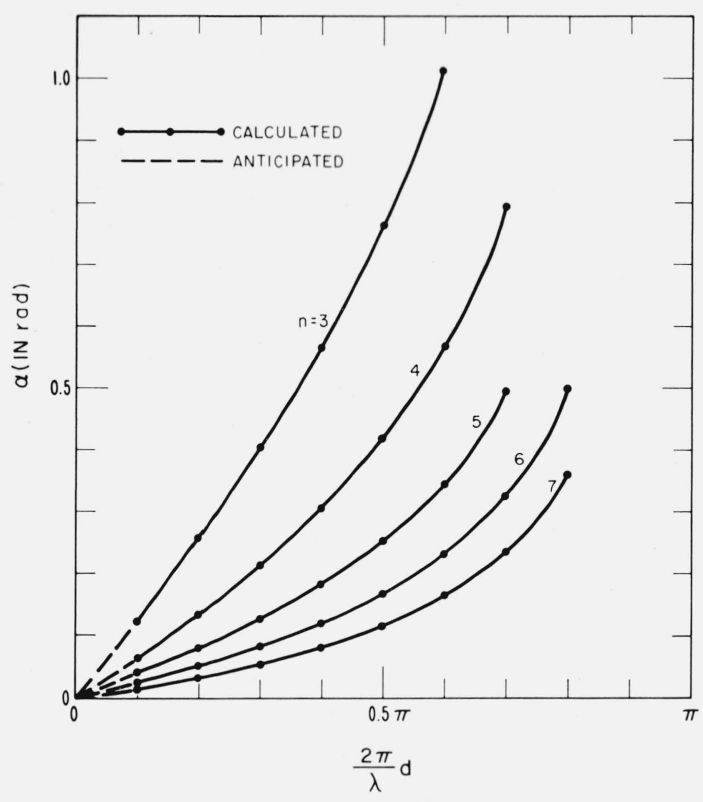

FigURE 7. Phases in radians required for optimum endfire arrays with equal sidelobes $(-20 \mathrm{~dB})$.
Ma, M. T., and D. K. Cheng (Apr. 1961b), A critical study of linear arrays with equal sidelobes, Syracuse Univ. Res. Inst. Report No. EE492-6104 T12.

Pritchard, R. L. (Jan. 1955), Discussion of DuHamel's paper, IRE Trans. Ant. Prop. AP-3, 40-43.

Reuss, M. L. (Feb. 1959), Some design considerations concerning linear arrays having Dolph-Tchebysheff amplitude distributions, U.S. Naval Res. Lab. Report No. 5240.

Rhodes, D. R. (June 1953), The optimum linear array for a single main beam, Proc. IRE 41, 793-794.

Riblet, H. J. (May 1947), Discussion of Dolph's paper, Proc. IRE 35 , 489-492.

Silver, S. (1949), Microwave Antenna Theory and Design, p. 581 (McGraw-Hill Book Co., New York, N.Y.).

Stearns, C. O. (1965), Computed performance of moderate size supergain endfire antennas, IEEE Trans. Ant. Prop. AP-13, No. 6.

Stegen, R. J. (Nov. 1960), Gain of Tchebysheff arrays, IRE Trans. Ant. Prop. AP-8, 629-631.

Tai, C. T. (Mar. 1963), On the optimum gain of uniformly spaced arrays of isotropic sources or dipoles, Ohio State Univ. Res. Report No. 1522-1.

Tai, C. T. (July 1964), The optimum directivity of uniformly spaced broadside arrays of dipoles, IEEE Trans. Ant. Prop. AP-12, $447-454$.

Taylor, T. T. (Sept. 1948), A discussion of the maximum directivity of an antenna, Proc. IRE 36, 1135.

Uzkov, A. I. (1946), An approach to the problem of optimum directive antenna design, Compt. Rend. (Doklady) Acad. Sci. URSS 3, 35.

Wilmotte, R. M. (July 1948), Note on practical limitations in the directivity of antennas, Proc. IRE 36, 878.

Yaru, N. (Sept. 1951), A note on supergain antenna arrays, Proc. IRE 39, 1081-1085.

(Paper 69D9-560) 